\title{
Modification of composition of a nanoemulsion with different cholesteryl ester molecular species: Effects on stability, peroxidation, and cell uptake
}

This article was published in the following Dove Press journal:

International Journal of Nanomedicine

13 September 2010

Number of times this article has been viewed

\author{
Cristina P Almeida' \\ Carolina G Vital' \\ Thais C Contente' \\ Durvanei A Maria ${ }^{2}$ \\ Raul C Maranhão ${ }^{1,3}$ \\ 'Lipid Metabolism Laboratory, \\ Heart Institute (InCor), Medical \\ School Hospital, ${ }^{2}$ Biochemistry and \\ Biophysics Laboratories, Butantan \\ Institute, ${ }^{3}$ Faculty of Pharmaceutical \\ Sciences, University of São Paulo, \\ São Paulo, Brazil
}

Purpose: Use of lipid nanoemulsions as carriers of drugs for therapeutic or diagnostic purposes has been increasingly studied. Here, it was tested whether modifications of core particle constitution could affect the characteristics and biologic properties of lipid nanoemulsions.

Methods: Three nanoemulsions were prepared using cholesteryl oleate, cholesteryl stearate, or cholesteryl linoleate as main core constituents. Particle size, stability, $\mathrm{pH}$, peroxidation of the nanoemulsions, and cell survival and uptake by different cell lines were evaluated.

Results: It was shown that cholesteryl stearate nanoemulsions had the greatest particle size and all three nanoemulsions were stable during the 237-day observation period. The $\mathrm{pH}$ of the three nanoemulsion preparations tended to decrease over time, but the decrease in $\mathrm{pH}$ of cholesteryl stearate was smaller than that of cholesteryl oleate and cholesteryl linoleate. Lipoperoxidation was greater in cholesteryl linoleate than in cholesteryl oleate and cholesteryl stearate. After four hours' incubation of human umbilical vein endothelial cells (HUVEC) with nanoemulsions, peroxidation was minimal in the presence of cholesteryl oleate and more pronounced with cholesteryl linoleate and cholesteryl stearate. In contrast, macrophage incubates showed the highest peroxidation rates with cholesteryl oleate. Cholesteryl linoleate induced the highest cell peroxidation rates, except in macrophages. Uptake of cholesteryl oleate nanoemulsion by HUVEC and fibroblasts was greater than that of cholesteryl linoleate and cholesteryl stearate. Uptake of the three nanoemulsions by monocytes was equal. Uptake of cholesteryl oleate and cholesteryl linoleate by macrophages was negligible, but macrophage uptake of cholesteryl stearate was higher. In H292 tumor cells, cholesteryl oleate showed the highest uptakes. HUVEC showed higher survival rates when incubated with cholesteryl stearate and smaller survival with cholesteryl linoleate H292 survival was greater with cholesteryl stearate.

Conclusion: Although all three nanoemulsion types were stable for a long period, considerable differences were observed in size, oxidation status, and cell survival and nanoemulsion uptake in all tested cell lines. Those differences may be helpful in protocol planning and interpretation of data from experiments with lipid nanoemulsions.

Keywords: emulsions, cholesterol, drug vehicles, lipoprotein receptors, solid lipid nanoparticles

\section{Introduction}

The use of nanoemulsions as drug delivery vehicles for the treatment of proliferative inflammatory diseases, such as cancer and atherosclerosis, has been increasingly studied. ${ }^{1-3}$ In search of more efficient drug delivery devices, the systematic modification of the composition of those nanoemulsion systems to optimize the pharmacologic properties of the drug-vehicle association is an important research pathway. 
In previous studies, we have been showing that cholesteryl ester-rich nanoemulsions resembling the structure of lipoproteins can function as drug-targeting devices ${ }^{4-6}$ to carry drugs to tumors ${ }^{7}$ or atheromatous lesions. ${ }^{8}$ Nonprotein nanoemulsions resembling low-density lipoproteins (LDL) can pick up apolipoprotein $\mathrm{E}$ (apo E) in contact with plasma and bind to LDL receptors using this apolipoprotein as a ligand. ${ }^{9}$ The properties of LDL or other lipoprotein-binding receptors are interesting in terms of drug delivery. Because lipids must be disposable for the building of new membranes required by the accelerated mitosis rates in many pathologic conditions, those receptors are frequently overexpressed, with endowment of a potent targeting mechanism. Those nanoemulsions have been constructed with cholesteryl oleate as a particle core and egg lecithin as the surface enveloping layer. Minor amounts of triolein and free cholesterol were included in the composition.

The aim of this study was to investigate whether the degree of saturation of the acyl radicals of cholesteryl esters that make up the nanoemulsion core may influence the physical and biologic properties of nanoemulsions. Substitution in the composition of the nanoemulsions of three different cholesteryl esters with the same acyl chain length, namely oleate (18:1), linoleate (18:2), and stearate (18:0), the chemical structures of which are shown in Figure 1, may influence the stability and peroxidation of nanoemulsion lipids and uptake by different cell lines. Survival of the cells upon coincubation with the three nanoemulsion cholesteryl esters was also examined. Results show that compositional changes in respect to cholesteryl ester molecular species can alter the biologic properties of the nanoemulsions.

\section{Methods \\ Materials}

The lipids were purchased from Sigma Chemical Company (St. Louis, MO). Cholesteryl [1-14C] oleate was purchased from Amersham (Little Chalfont, Buckinghamshire, UK). All the chemicals and solvents used in the study were of analytic grade and purchased from Sigma Chemical Company.

\section{Preparation of artificial nanoemulsions}

The artificial nanoemulsions were prepared according to the method described by Ginsburg et $\mathrm{al}^{10}$ and modified by Maranhão et al. ${ }^{11} \mathrm{~A}$ lipid mixture composed of $40 \mathrm{mg}$ cholesteryl oleate, $20 \mathrm{mg}$ egg phosphotidylcholine, $1 \mathrm{mg}$ triolein, and

A

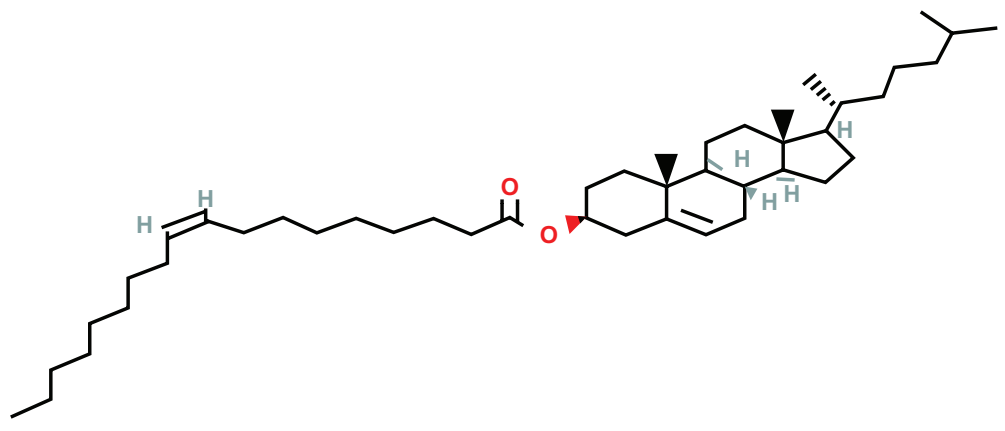

B

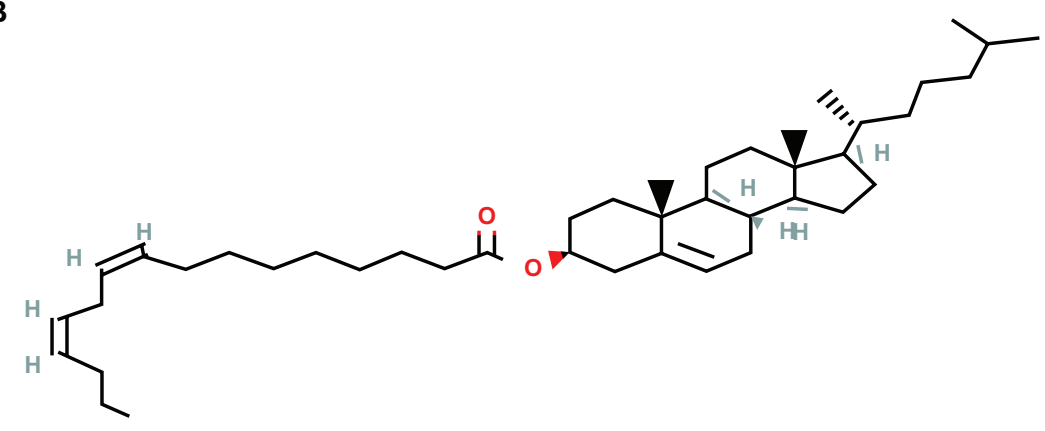

C

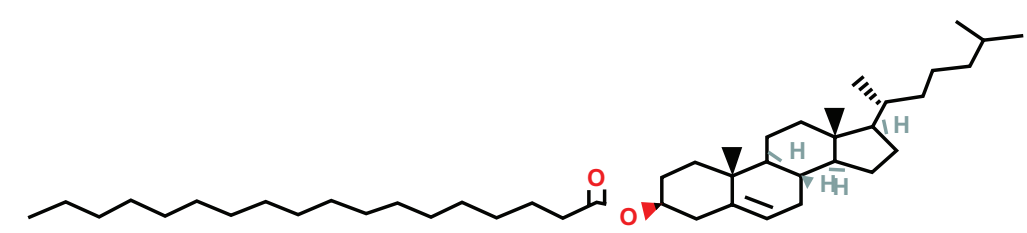

Figure I Chemical structure of the cholesteryl esters used in the making of the nanoemulsions: A) Cholesteryl oleate (I8:I). B) Cholesteryl linoleate (I8:2). C) Cholestery| stearate $(18: 0)$. 
$0.5 \mathrm{mg}$ free cholesterol was dissolved in chloroform:methanol (2:1) and dispensed into vials. For the nanoemulsion with different cholesteryl ester species, in place of cholesteryl oleate, we used cholesteryl linoleate or cholesteryl stearate. The mixture was dried under a nitrogen stream followed by overnight vacuum desiccation at $4^{\circ} \mathrm{C}$ to remove residual solvents. The dried lipids were resuspended in $10 \mathrm{~mL}$ of tris- $\mathrm{HCl} 0.01 \mathrm{M}$ buffer at $\mathrm{pH} 8.0$ and the suspension was emulsified by prolonged ultrasonic irradiation for 180 minutes in a nitrogen atmosphere and with a controlled temperature. The emulsified lipid suspension was then transferred to clean tubes and ultracentrifugated at $195,000 \mathrm{~g}$ for 30 minutes at $4^{\circ} \mathrm{C}$. The supernatant was removed by aspiration with a needle. Solid $\mathrm{KBr}$ was added to the remaining solution for density adjustment to $1.21 \mathrm{~g} / \mathrm{mL}$. A second ultracentrifugation step was then performed at 195,000 $\mathrm{g}$ for 120 minutes at $4^{\circ} \mathrm{C}$. The nanoparticles were recovered by aspiration and dialyzed overnight in tris- $\mathrm{HCl}$ buffer at a $\mathrm{pH}$ 8.0 for $\mathrm{KBr}$ removal. The nanoemulsion was passed through a $0.22 \mu \mathrm{m}$ Millipore ${ }^{\mathrm{TM}}$ filter (Millipore Inc., Billerica, MA). Trace amounts of cholesteryl $\left[1-{ }^{14} \mathrm{C}\right]$ oleate were added to the initial solution.

\section{Composition of nanoemulsion formulations}

The composition of cholesteryl oleate, cholesteryl linoleate, and cholesteryl stearate nanoemulsion was determined after lipid extraction by the Folch method, resolution of the lipid components by thin-layer chromatography, iodine staining, and determination of the density of bands using a model L.PIX transluminator (Loccus, São Paulo, Brazil).

\section{Particle size, polydispersity, and zeta potential analysis}

Each batch of nanoemulsions was characterized for particle size, polydispersity, and zeta potential by the dynamic light scattering method using a ZetaPALS Zeta Potential Analyzer (Brookhaven Instruments, Holtsville, NY). For size measurement, nanoemulsion samples $(35 \mu \mathrm{L})$ were diluted in $2.0 \mathrm{~mL}$ tris- $\mathrm{HCl} 0.01 \mathrm{M}$ buffer at $\mathrm{pH} 8.05$, ie, the same buffer that was used in the making of the nanoemulsions. At the time of particle size determination, average count rate was maintained between 50-200 kcps to achieve reproducibility. The mean effective hydrodynamic diameter of the oil droplets on log-normal size distribution mode was considered for comparative evaluation. For zeta potential measurements, the nanoemulsion samples $(45 \mu \mathrm{L})$ were diluted to $1.5 \mathrm{~mL}$ of $\mathrm{KCl} 1.0 \mathrm{mM}$. Hydrodynamic diameter, polydispersity, and zeta potential were reported as the average of two measurements performed at $25^{\circ} \mathrm{C}$. For stability studies, all samples were kept at $4^{\circ} \mathrm{C}$ over a period of 240 days, and particle size and phase Doppler interferometry measurements were undertaken every 15 days. Zeta potential measurements were performed on days 1 and 210 after manufacture.

\section{$\mathrm{pH}$ analysis}

Measurements of the $\mathrm{pH}$ of the nanoemulsions were performed using a digital $\mathrm{pH}$ meter (Digimed, São Paulo, Brazil). For stability studies, all samples were kept at $4^{\circ} \mathrm{C}$ over a period of 240 days and the $\mathrm{pH}$ measurements were undertaken every 15 days.

\section{Lipid peroxidation analysis}

The lipid peroxidation of each nanoemulsion was measured by the thiobarbituric acid reactive substances (TBARS) method. ${ }^{12}$ For stability studies, all samples were kept at $4{ }^{\circ} \mathrm{C}$ over a period of 240 days and the lipid peroxidation measurements were undertaken every 15 days.

\section{Cell uptake in the nanoemulsions}

Human umbilical vein endothelial cells (HUVEC) were cultured in M-199 medium containing 20\% fetal bovine serum. H292 (mucoepidermoid pulmonary carcinoma) and THP-1 (a human monocytic leukemia cell line), referred to here as monocytes and fibroblasts, were cultured in RPMI-1640 containing 20\% fetal bovine serum and all cells were used from passages four or five. The THP-1-monocytes was differentiated in THP-1-macrophages using $50 \mathrm{nM}$ of phorbol 12-myristate 13-acetate (PMA; Sigma-Aldrich) by 24 hours. The cells were plated at $5 \times 10^{5}$ grown confluence, and $4 \mathrm{~mL}$ of medium with fetal bovine serum were added followed by 24 hours of incubation at $36^{\circ} \mathrm{C}$. After 24 hours, the medium was changed to medium lipoprotein-deficient serum to overexpress the receptors. After 24 hours of incubation, lipoprotein-deficient serum medium was substituted with medium containing fetal bovine serum. All cell lines were incubated with cholesteryl oleate, cholesteryl linoleate, and cholesteryl stearate in concentrations ranging from $0.15 \mathrm{mg} / \mathrm{mL}$ to $5.0 \mathrm{mg} / \mathrm{mL}$ for four hours. At the end of incubation, the supernatant was collected for TBARS analyses. The cells were washed three times with phosphate-buffered saline. The cells were harvested from the culture dish by treatment with a trypsin solution $0.02 \%$ and centrifuged for five minutes at $4^{\circ} \mathrm{C}$. The cell pellets were sonicated and stored at tubes with scintillation solution (Packard BioScience, Groeningen, The Netherlands). The cell uptake was measured by radioactive counting of the cell lysate in a scintillation solution using a Packard 1600 TR model 
Liquid Scintillation Analyzer (Palo Alto, CA). The protein concentration was determined by Bradford assay. ${ }^{13}$

\section{Lipid peroxidation of the nanoemulsion-cell incubates}

After four hours of cholesteryl oleate, cholesteryl linoleate, and cholesteryl stearate nanoemulsion incubation with cells, $100 \mu \mathrm{L}$ of solely culture medium was collected for lipid peroxidation analysis ${ }^{14}$ by the TBARS method. ${ }^{12}$

\section{Cell survival upon incubation with nanoemulsions}

The HUVEC and H292 cell lines were incubated with cholesteryl oleate, cholesteryl linoleate, and cholesteryl stearate for four hours in 96-well plates at concentrations ranging from $0.15 \mathrm{mg} / \mathrm{mL}$ to $5.0 \mathrm{mg} / \mathrm{mL}$. The number of viable cells was estimated by the MTT assay (3-[4,5-dimethylthiazol2-yl)-2,5-diphenyltetrazolium-bromide ${ }^{15}$ (Sigma Aldrich). The cell survival data were plotted as dose-response curves with nanoemulsion concentrations. The incubation time was 24 hours, after which the medium was replaced with growth medium after washing of cells using phosphate-buffered saline. After 24 hours of incubation, $25 \mu \mathrm{L}$ of MTT dye was added to each well and the cells were incubated for a further three hours at $37^{\circ} \mathrm{C}$. Then the medium was removed and $100 \mu \mathrm{L}$ dimethyl sulfoxide was added to each well. The plates were shaken for 30 seconds and the absorbance was recorded at $540 \mathrm{nM}$ using a microplate reader.

a)

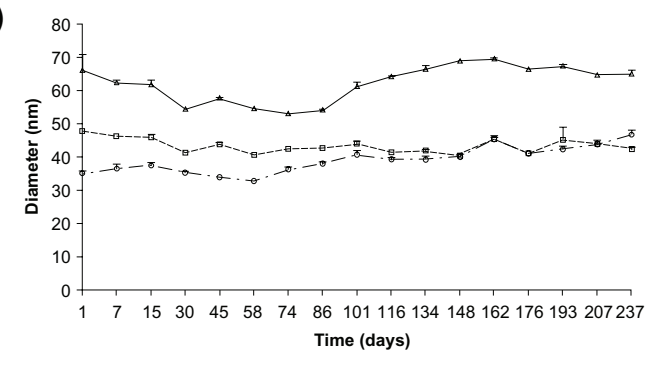

c)

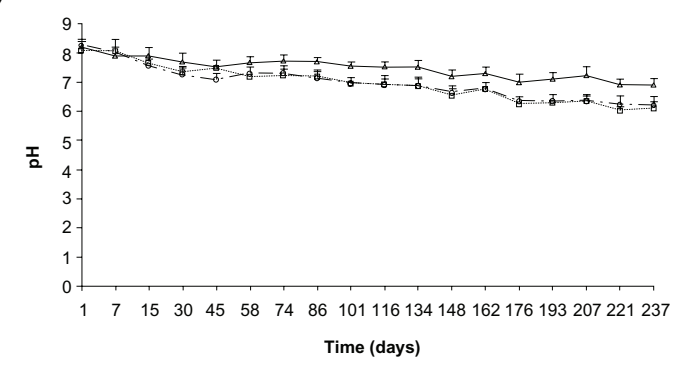

\section{Results}

\section{Composition of the nanoemulsion formulations}

Prepared as described, cholesteryl oleate had 67\% phospholipids, $31 \%$ cholesteryl esters, $1 \%$ free cholesterol, and $1 \%$ triglycerides. Cholesteryl stearate had $69 \%$ phospholipids, $29 \%$ cholesteryl esters, $1 \%$ free cholesterol, and $1 \%$ triglycerides. Cholesteryl linoleate had $72 \%$ phospholipids, $25 \%$ cholesteryl esters, $1 \%$ free cholesterol, and $2 \%$ triglycerides.

\section{Particle size, $\mathrm{pH}$ lipid peroxidation, and zeta potential}

Figure 2 shows the characteristics of all the nanoemulsions. The sizes of all three preparations were stable during the 237-day observation period, although cholesteryl stearate showed greater size variations than the other two preparations. Cholesteryl stearate nanoemulsions had the greatest particle size, as shown in Figure 2A. As shown in Figure 2B, polydispersity of the cholesteryl stearate particles tended to decrease over time, whereas polydispersity of both cholesteryl oleate and cholesteryl linoleate was unchanged. Figure 2C shows that, during the 237-day observation period, the $\mathrm{pH}$ of the three nanoemulsion preparations tended to decrease over time, but the $\mathrm{pH}$ fall of cholesteryl stearate was smaller than that occurring for cholesteryl oleate and cholesteryl linoleate. As shown in Figure 2D, lipid peroxidation was initially greater in cholesteryl linoleate than in cholesteryl oleate and cholesteryl stearate. Subsequently, there was further

b)

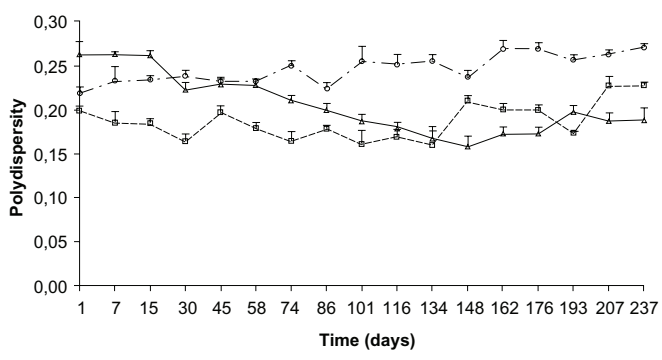

d)

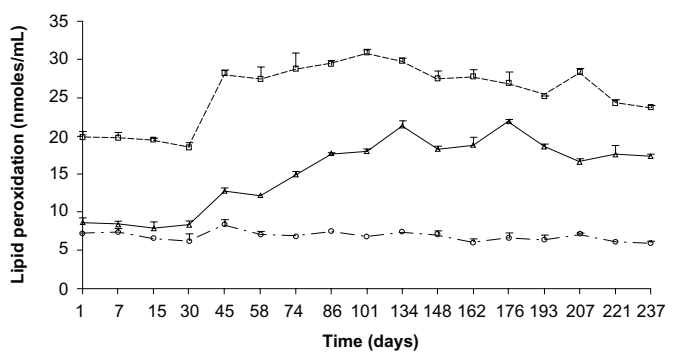

Figure 2 Stability of the three different nanoemulsions, CO (circles), CL (triangles), and CS (squares), as observed during 237 days, in respect to a) size, b) polydispersity, c) $\mathrm{pH}$, and d) lipid peroxidation evaluated by the TBARS method. Data are expressed as means \pm SD of two samples.

Abbreviations: $\mathrm{CO}$, cholesteryl oleate; $\mathrm{CL}$, cholesteryl linoleate; CS, cholesteryl stearate; SD, standard deviation; TBARS, thiobarbituric acid reactive substances. 
peroxidation in cholesteryl linoleate, whereas peroxidation markedly increased in cholesteryl stearate, and was unchanged over time for cholesteryl oleate.

When measured on day 1 , the zeta potential was the same in the cholesteryl oleate and cholesteryl stearate preparations $(-32.6 \pm 2.2 \mathrm{mV}$ and $-33.9 \pm 2.4 \mathrm{mV}$, respectively) and tended to decrease on day $210(-44.0 \pm 1.8 \mathrm{mV}$ and $-38.2 \pm 1.6 \mathrm{mV})$. For cholesteryl linoleate, the zeta potential on day $1(-16.0 \pm 3.2 \mathrm{mV})$ was higher than for cholesteryl oleate and cholesteryl stearate, and also tended to decrease on day $210(-40.0 \pm 3.0 \mathrm{mV})$.

\section{Cell lipid peroxidation in incubates with nanoemulsions}

As shown in Figure 3A, lipid peroxidation in the HUVEC incubates was minimal in the presence of cholesteryl oleate and more pronounced by increasing the amounts of cholesteryl linoleate and cholesteryl stearate. Similar results were observed in fibroblasts with minimal peroxidation when cholesteryl oleate was added (Figure 3B). In monocytes, peroxidation was minimal, not only in the presence of cholesteryl oleate, but also for cholesteryl stearate (Figure 3C). In the neoplastic $\mathrm{H} 292$ cells, peroxidation was also lower with cholesteryl oleate (Figure 3E). In contrast, the highest peroxidation rates with addition of cholesteryl oleate was in macrophage cells (Figure 3D). It is noteworthy that cholesteryl linoleate induced the highest cell peroxidation rates with the exception of that for the macrophage incubate.

\section{Cell uptake in the nanoemulsions}

In Figure 4A, it is shown that the cholesteryl oleate uptake by HUVEC was markedly greater than that of cholesteryl linoleate and cholesteryl stearate, seen from $0.15 \mathrm{mg} / \mathrm{mL}$ concentration and increasing until $4.14 \mathrm{mg} / \mathrm{mL}$, when an apparent plateau was reached. Cholesteryl oleate uptake by fibroblasts was also greater than that of the other nanoemulsions, as shown in Figure 4B. In contrast, uptake of the three nanoemulsions by monocytes was similar until a concentration of $2.2 \mathrm{mg} / \mathrm{mL}$ nanoemulsion lipids was reached and, henceforth, the cholesteryl oleate uptake was clearly lower than that of the two other nanoemulsion types (Figure 4C). With regard to the macrophage cells, uptake of both cholesteryl oleate and cholesteryl linoleate was negligible at all concentration points, whereas the uptake of cholesteryl stearate was continuously increased (Figure 4D). Finally, in the neoplastic H292 cell lineage, cholesteryl oleate was taken up in greater amounts than cholesteryl linoleate and cholesteryl stearate until $4.14 \mathrm{mg} / \mathrm{mL}$, when the uptake of all three was equal (Figure 4E).

\section{Cell survival upon incubation with nanoemulsions}

After four hours of incubation with increasing amounts of the three nanoemulsion types, $\mathrm{HUVEC}\left(\mathrm{IC}_{50} 1.15-4.14 \mathrm{mg} / \mathrm{mL}\right.$ for cholesteryl oleate, nearly $1.15 \mathrm{mg} / \mathrm{mL}$ for cholesteryl linoleate, and $4.14-5.0 \mathrm{mg} / \mathrm{mL}$ for cholesteryl stearate) showed a greater survival rate when incubated with cholesteryl stearate and
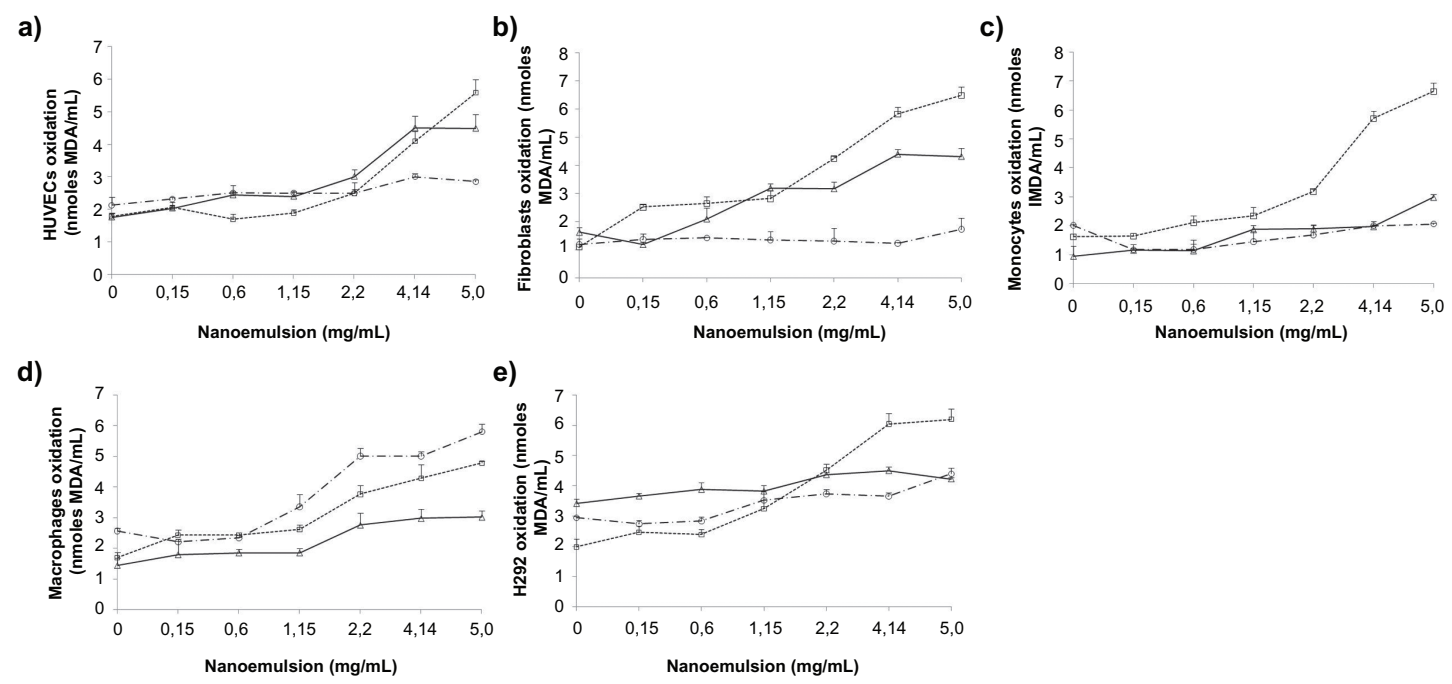

Figure 3 Lipid peroxidation measured by the TBARS method in incubates of a) HUVEC, b) fibroblasts, c) monocytes, d) macrophages, and e) H292 cells with increasing concentrations of CO (circles), CL (triangles), and CS (squares) nanoemulsions. Lipid peroxidation was measured in $100 \mu \mathrm{L}$ of the culture media after four hours of incubation. Control corresponds to point zero, in which there was no addition of nanoemulsion to the cells. Data are expressed as means \pm SD of three experiments. Abbreviations: CO, cholesteryl oleate; CL, cholesteryl linoleate; CS, cholesteryl stearate; SD, standard deviation; TBARS, thiobarbituric acid reactive substances; HUVEC, human umbilical vein endothelial cells. 
a)

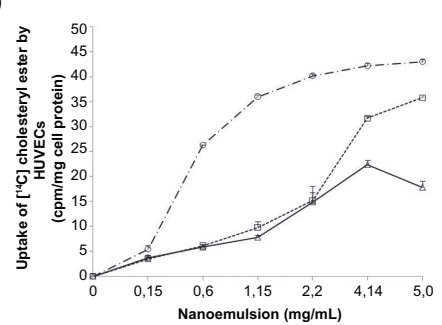

d)

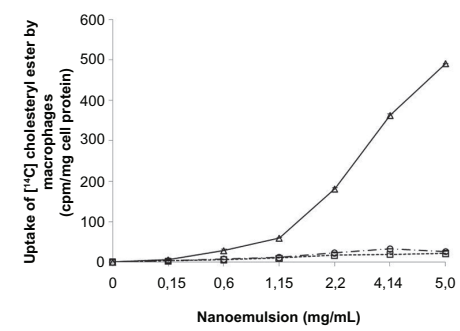

b)

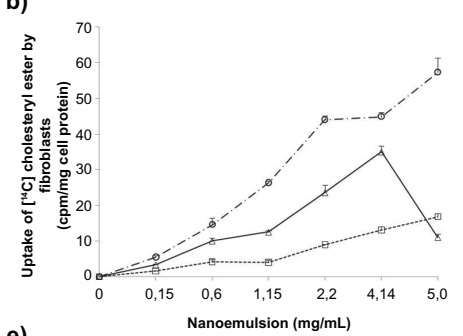

e)

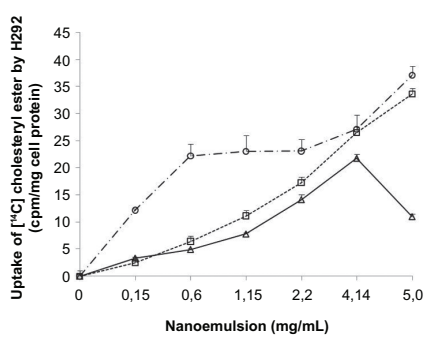

c)

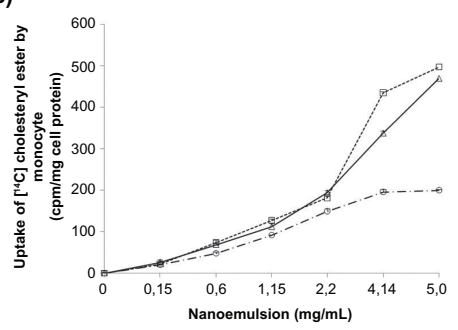

Figure 4 Uptake by a) HUVEC, b) fibroblasts, c) monocytes, d) macrophages, and e) H292 cells of increasing amounts of CO (circles), CL (triangles), and CS (squares) after four hours of incubation of radioactively labeled nanoemulsions with the cultured cells at $37^{\circ} \mathrm{C}$. Data are expressed as means \pm SD of three experiments.

Abbreviations: CO, cholesteryl oleate; CL, cholesteryl linoleate; CS, cholesteryl stearate; SD, standard deviation; HUVEC, human umbilical vein endothelial cells.

shorter survival with cholesteryl linoleate (Figure 5A). For the neoplastic $\mathrm{H} 292$ cells $\left(\mathrm{IC}_{50}>1.15 \mathrm{mg} / \mathrm{mL}\right.$ for cholesteryl oleate, $<4.14 \mathrm{mg} / \mathrm{mL}$ for cholesteryl linoleate, and nearly $5.0 \mathrm{mg} / \mathrm{mL}$ for cholesteryl stearate), survival rates were greater when incubated with cholesteryl stearate, mainly at higher nanoemulsion concentrations (Figure 5B).

\section{Discussion}

Lipid nanoparticles have been increasingly perceived as systems that can be produced in cost-effective, large-scale quantities, ${ }^{16}$ and clinical trials have already been performed that show the advantages of these systems. Among the advantages when nanoparticles are used as drug carriers is a marked reduction in toxicity of the chemotherapeutic agents involved. ${ }^{17,18}$ This highlights the importance of expanding our knowledge of the various technologic and biologic aspects of lipid nanoparticles, eg, how changes in chemical composition determine size, charge, stability, and uptake by different cell lines. ${ }^{19,20}$

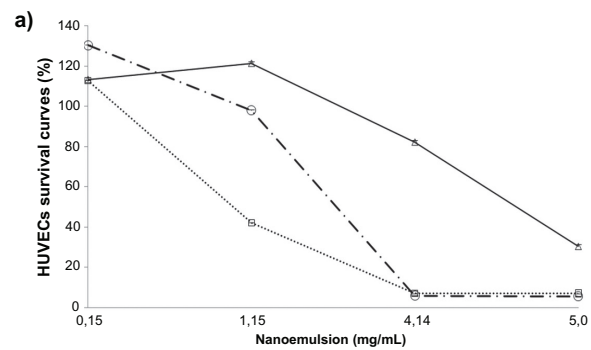

In this study, the cholesteryl stearate nanoemulsion showed a greater particle size than the other two nanoemulsion preparations. The greater hydrophobicity of cholesteryl stearate, together with its stereochemical properties, may have facilitated the formation of larger particle cores.

The substitution of three different cholesteryl esters in the nanoemulsion composition did not affect the stability of the nanoemulsion particles, in that all three types showed stable particle sizes for approximately eight months without addition of antioxidant agents. Presumably, the association of drugs with the nanoemulsion structures would be an important factor in terms of both the size and stability of the formed nanoemulsion-drug particles. In this setting, it is difficult to foresee whether cholesteryl stearate composition will also favor the formation of larger nanoemulsion-drug particles.

The $\mathrm{pH}$ of the cholesteryl linoleate and cholesteryl oleate nanoemulsions tended to decrease continuously over the eight-month observation period. The decrease in $\mathrm{pH}$ was

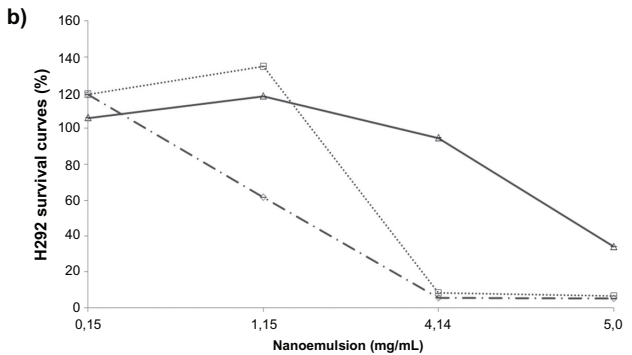

Figure 5 Survival curves of a) HUVEC and b) H292 cells 24 hours after incubation with CO (circles), CL (triangles), and CS (square) nanoemulsions, as evaluated by the MTT method. Data are expressed as means \pm SD of three experiments.

Abbreviations: $\mathrm{CO}$, cholesteryl oleate; $\mathrm{CL}$, cholesteryl linoleate; $\mathrm{CS}$, cholesteryl stearate; SD, standard deviation; HUVEC, human umbilical vein endothelial cells. 
somewhat less for cholesteryl stearate than for the two other preparations. The peroxidation of the nanoemulsion lipids that occurred in all three preparations over the eight-month period might have contributed to the decreased $\mathrm{pH}$.

Lipid peroxidation monitored by TBARS was greater for cholesteryl linoleate than for the other preparations from day 1 onwards, tending to increase further after day 30 of the study period. Because polyunsaturated acyl chains are more prone to peroxidation than saturated chains, this finding was indeed expected. Although peroxidation was low for both cholesteryl oleate and cholesteryl stearate soon after manufacture, peroxidation for cholesteryl stearate increased markedly more than for cholesteryl oleate after day 30 . This would be unexpected in view of the chain saturation. Since the nanoemulsions are a quaternary system, interactions with the other components, ie, phosphatidylcholine, triolein, and free cholesterol, may have accounted for those results. In future studies, a more detailed peroxidation profile should be performed, due to the limitations of the TBARS method for evaluation of peroxidation status, such as underestimation of total peroxidation and lack of discrimination between primary and secondary peroxidation products. Mass spectrometry-based and other methods also have the ability to distinguish peroxidation of the different lipid molecular species specifically.

The zeta potential is a key factor in evaluation of the stability of colloidal systems. The negative zeta potential value would allow prediction of good colloidal stability due to the high-energy barrier between particles..$^{21}$ Thus, zeta potential results suggest that all three nanoemulsion preparations were stable on day 1 and remained stable at 210 days.

According to the cell uptake results, it is remarkable that the cholesteryl oleate nanoemulsion showed the highest uptake rates in three of the five tested cell lines, namely HUVEC, fibroblasts, and H292. Cholesteryl oleate had the smallest particles 24 hours after manufacture, what suggests that particle size can facilitate cell uptake. On the other hand, cholesteryl stearate showed the largest particles and the greatest uptake rates by the THP-1 lineage differentiated in macrophages by PMA. This suggests that larger particles are removed by scavenger receptors rather than by lipoprotein receptors; the uptake by macrophages of the smaller nanoemulsion particles. Cholesteryl oleate and cholesteryl linoleate uptake was negligible. In fact, THP-1 macrophages show that expression of scavenger receptors and uptake of cholesteryl esters by those cells is greater than by primary culture macrophages. ${ }^{22}$

It is worthwhile to point out that uptake of the three nanoemulsion types by the monocyte lineage was several-fold greater than that of HUVEC, fibroblasts, and H292 cells. Because this cell lineage is of neoplastic origin, it is possible that overexpression of lipoprotein receptors associated with neoplasias had occurred, leading to the increased uptake. In this regard, neoplasia-associated LDL receptor overexpression can be as high as one hundred times that of normal cells, as documented in acute myelocytic leukemia. ${ }^{23,24}$

The experiment for cell peroxidation showed that cholesteryl linoleate induced greater oxidation in HUVEC, fibroblasts, monocytes, and $\mathrm{H} 292$ cell lines. This is conceivably linked with the greater number of double bonds in this cholesteryl ester molecular species. ${ }^{25}$ Interestingly, macrophages were the only cell line in which cholesteryl oleate nanoemulsions, but not cholesteryl linoleate, produced the highest peroxidation rates. Macrophages internalize only minimal amounts of cholesteryl oleate nanoemulsions, which suggests that uptake of the nanoemulsion is not a precondition for peroxidation to occur.

As tested in two cell lines, HUVEC and H292, cell survival was longer in incubates with cholesteryl stearate, compared with cholesteryl linoleate and cholesteryl oleate. This finding is in agreement with those from other investigators ${ }^{26}$ that showed a clear-cut trend for small nanoparticles to be more toxic to cells than larger ones. Interestingly, the uptake of cholesteryl stearate nanoemulsion by both cell lines was the lowest among the three tested nanoemulsions. This would suggest that perhaps larger lipid uptakes would damage cells.

In the in vivo studies, reduction in toxicity of several chemotherapeutic agents, including paclitaxel, ${ }^{5}$ etoposide, ${ }^{27}$ carmustine, ${ }^{4}$ and daunorubicin, ${ }^{28}$ incorporated into the nanoemulsion was remarkable. Those data support the validity of lipid nanoemulsion systems as drug vehicles in therapeutics.

\section{Conclusion}

Our results showed that all three nanoemulsion preparations were stable for a long period of time. The cholesteryl linoleate nanoemulsion was more prone to lipid peroxidation and, in general terms, promoted greater cell peroxidation at higher concentrations. Cholesteryl stearate nanoemulsions showed high macrophage uptake, probably related to larger particle size. Cholesteryl oleate showed greatest uptakes by HUVEC, fibroblasts, and H292 cells, with low peroxidation levels. The different functional characteristics of mono-, poly-, and saturated cholesteryl esters in terms of size, stability, peroxidation, cell uptake, and survival documented in this study may provide strategic elements for designing study protocols using lipid nanoemulsions for treatment or diagnosis of several diseases. 


\section{Acknowledgments}

This study was supported by Fundação do Amparo à Pesquisa do Estado de São Paulo (FAPESP), São Paulo, Brazil. Dr Maranhão has a research award from Conselho Nacional de Desenvolvimento Científico e Tecnológico, Brasília, Brazil, and Dr Almeida had a scholarship from FAPESP. The authors are grateful to Dr Francisco R Laurindo from the Laboratory of Vascular Biology at the Heart Institute, Medical Faculty, University of São Paulo, Brazil, for his generous donation of the THP-1 cells used in this study, and to Dr Durvanei A Maria from the Biochemistry and Biophysics Laboratories, Butantan Institute, São Paulo, Brazil, for support and generous donation of the fibroblast cells.

\section{Disclosure}

The authors report no conflicts of interest in this work.

\section{References}

1. Maranhão RC, Garicochea B, Silva EL, et al. Plasma kinetics and biodistribution of a lipid emulsion resembling low-density lipoprotein in patients with acute leukemia. Cancer Res. 1994;54:4660-4666.

2. Carmona-Ribeiro AM. Biomimetic nanoparticles: Preparation, characterization and biomedical applications. Int J Nanomedicine. 2010;5: 249-259.

3. Maranhão RC, Tavares ER, Padoveze AF, Valduga CJ, Rodrigues DG, Pereira MD. Paclitaxel associated with cholesterol-rich nanoemulsions promotes atherosclerosis regression in the rabbit. Atherosclerosis. 2008; 197:959-966.

4. Maranhão RC, Graziani SR, Yamaguchi N, et al. Association of carmustine with a lipid emulsion: In vitro, in vivo and preliminary studies in cancer patients. Cancer Chem Pharmacol. 2002;49:487-498.

5. Pires LA, Hegg R, Valduga CJ, Graziani SR, Rodrigues DG, Maranhão RC. Use of cholesterol-rich nanoparticles that bind to lipoprotein receptors as a vehicle to paclitaxel in the treatment of breast cancer: Pharmacokinetics, tumor uptake and a pilot clinical study. Cancer Chemother Pharmacol. 2009;63:281-287.

6. Dias ML, Carvalho JP, Rodrigues DG, Graziani SR, Maranhão RC. Pharmacokinetics and tumor uptake of a derivatized form of paclitaxel associated to a cholesterol-rich nanoemulsion (LDE) in patients with gynecologic cancers. Cancer Chemother Pharmacol. 2007;59: 105-111.

7. Graziani SR, Igreja FAF, Hegg R, et al. Uptake of a cholesterol rich emulsion by breast cancer. Gynecol Oncol. 2002;85:493-497.

8. Saremback IJ. From systemic shotgun to site-specific nanoparticletargeted delivery: A new paradigm for drug delivery. Arterioscler Thromb Vasc Biol. 2008;28:1879-1881.

9. Hirata RD, Hirata MH, Mesquita CH, Cesar TB, Maranhao RC. Effects of apolipoprotein B-100 on the metabolism of a lipid microemulsion model in rats. Biochim Biophys Acta. 1999;1437:53-62.
10. Ginsburg GS, Small DM, Atkinson D. Microemulsions of phospholipids and cholesterol esters: Protein-free models of low density lipoprotein. J Biol Chem. 1982;257:8216-8227.

11. Maranhão RC, Cesar TB, Pedroso-Mariani SR, Hirata MH, Mesquita CH. Metabolic behavior in rats of a nonprotein microemulsion resembling low-density lipoprotein. Lipids. 1993;28:691-696.

12. Ohkawa H, Ohishi N, Yagi K. Assay for lipid peroxides in animal tissues by thiobarbituric acid reaction. Anal Biochem. 1979;95:351-358.

13. Bradford MM. A rapid and sensitive method for the quantitation of microgram quantities of protein utilizing the principle of protein-dye binding. Anal Biochem. 1976;72:248-254.

14. Fuhrman B, Judith O, Keidar S, Ben-Yaish L, Kaplan M, Aviram M. Increased uptake of LDL by oxidized macrophages is the result of an initial enhanced LDL receptor activity and of a further progressive oxidation of LDL. Free Radic Biol Med. 1997;23:34-46.

15. Marks DC, Belov L, Davey MW, Davey RA, Kidman AD. The MTT cell viability assay for cytotoxicity testing in multidrug-resistant human leukemic cells. Leuk Res. 1992;16:1165-1173.

16. Kaur IP, Bhandari R, Bhandari S, Kakkar V. Potential of solid lipid nanoparticles in brain targeting. J Control Release. 2008;127:97-109.

17. Manjunath K, Reddy JS, Venkateswarlu V. Solid lipid nanoparticles as drug delivery systems. Methods Find Exp Clin Pharmacol. 2005; 27:127-144.

18. Ma P, Dong X, Swadley CL, et al. Development of idarubicin and doxorubicin solid lipid nanoparticles to overcome Pgp-mediated multiple drug resistance in leukemia. J Biomed Nanotechnol. 2009;5: 151-161.

19. Desai MP, Labhasetwar-Elke V, Amidon D, et al. The mechanism of uptake of biodegradable microparticles in Caco-2 cells is size dependent. Pharm Res. 1997;14:1568-1573.

20. Schroeder A, Levins CG, Cortez C, Langer R, Anderson DG. Lipid-based nanotherapeutics for siRNA delivery. J Intern Med. 2010;267:9-21.

21. Mora-Huertas CE, Fessi H, Elaissari A. Polymer-based nanocapsules for drug delivery. Int J Pharm. 2010;385:113-142.

22. Banka CL, Black AS, Dyer CA, Curtiss LK. THP-1 cells form foam cells in response to coculture with lipoproteins but not platelets. J Lipid Res. 1991;32:35-43.

23. Ho YK, Smith RG, Brown MS, Goldstein JL. Low-density lipoprotein (LDL) receptor activity in human acute myelogenous leukemia cells. Blood. 1978;52:1099-1114.

24. Vitols S, Gahrton G, Ost A, Peterson C. Elevated low density lipoprotein receptor activity in leukemic cells with monocytic differentiation. Blood. 1984;63:1186-1193.

25. Krieger M, Brown MS, Faust J, Goldstein JL. Replacement of endogenous cholesteryl ester of low density lipoprotein with exogenous cholesteryl linoleate. J Biol Chem. 1978;253:4093-4101.

26. de Jong WH, Borm PJ. Drug delivery and nanoparticles: Applications and hazards. Int J Nanomedicine. 2008;3:133-149.

27. Lo Prete AC, Maria DA, Rodrigues DG, Valduga CJ, Ibañez OC, Maranhão RC. Evaluation in melanoma-bearing mice of an etoposide derivative associated to a cholesterol-rich nano-emulsion. J Pharm Pharmacol. 2006;58:801.

28. Teixeira RS, Valduga CJ, Benvenutti LA, Schreier S, Maranhão RC. Delivery of daunorubicin to cancer cells with decreased toxicity by association with a lipidic nanoemulsion that binds to LDL receptors. J Pharm Pharmacol. 2008;60:1287-1295.
International Journal of Nanomedicine

\section{Publish your work in this journal}

The International Journal of Nanomedicine is an international, peerreviewed journal focusing on the application of nanotechnology in diagnostics, therapeutics, and drug delivery systems throughout the biomedical field. This journal is indexed on PubMed Central, MedLine, CAS, SciSearch $\AA$, Current Contents $₫ /$ Clinical Medicine,

\section{Dovepress}

Journal Citation Reports/Science Edition, EMBase, Scopus and the Elsevier Bibliographic databases. The manuscript management system is completely online and includes a very quick and fair peer-review system, which is all easy to use. Visit http://www.dovepress.com/ testimonials.php to read real quotes from published authors. 